\title{
Relación entre los niveles de compuestos volátiles sulfurados con los niveles de RANKL y OPG en pacientes con periodontitis crónica moderada o severa: Estudio transversal
}

\author{
Relationship between the levels of volatile sulfur compounds with \\ the levels of RANKL and OPG in patients with moderate or severe \\ periodontitis: A cross sectional study
}

\author{
Prieto Damm D 1 , Sanz Ruiz A¹, Chaparro Padilla A ${ }^{1}$, Inostroza Silva C², Ramírez Lobos V³
}

\begin{abstract}
RESUMEN
Antecedentes: El efecto de los Compuestos Volátiles Sulfurados (CVS) sobre los tejidos periodontales, específicamente a nivel del eje RANKL/OPG, no ha sido dilucidado y en la actualidad existe escasa literatura al respecto publicada. Objetivo: Evaluar si los CVS medidos en la cavidad oral de pacientes con periodontitis crónica moderada a severa se relacionan con la expresión de RANKL y OPG a nivel de fluido gingival crevicular (FGC). Método: Se realizó un estudio transversal con 71 pacientes derivados de la Unidad de Diagnóstico de la Clínica Odontológica Docente Asistencial de la Universidad de los Andes. Posterior a la realización de un examen periodontal completo se tomaron muestra de los niveles de CVS de la boca de los pacientes mediante un monitor de sulfuros y muestras de FGC para evaluar los niveles de RANKL y OPG mediante test de ELISA. Los datos obtenidos fueron analizados mediante test de correlación de Spearman. Resultados: Al evaluar la correlación de los niveles de CVS con los niveles de RANKL, OPG y la razón RANKL/OPG, se observó un $R$ de 0.098 con un $p$ value $=0.41 ;-0.084$ con un $p$ value $=0.48$ y 0.067 con un $p$ value $=0.57$ respectivamente. Conclusiones: El presente estudio no pudo demostrar si existe una relación entre los niveles de CVS con la expresión de RANKL y OPG en el FGC de pacientes con periodontitis crónica.
\end{abstract}

Rev. Clin. Periodoncia Implantol. Rehabil. Oral Vol. 6(1); 20-24, 2013.

Palabras clave: Halitosis, periodontitis, compuestos volátiles sulfurados, RANKL y OPG.

\begin{abstract}
Background: The effect of the Volatile Sulfur Compounds (VSC) on the periodontal tissues, specifically at the RANKL/OPG level has not been elucidate and there is little literature published on this subject. Aim: To explore if the VSC levels measured in the oral cavity of patients with moderate or severe chronic periodontitis are correlated with the expression of RANKL and OPG at the gingival fluid level (GF). Method: A crosssectional study was conducted with 71 patients referred by the Universidad de los Andes's dentistry diagnosis department. After undergoing fullmouth periodontal exam, levels of CVS were recorded using a sulfur monitor and then GF was extracted to assess RANKL and OPG levels with ELISA. Data was analyzed with Spearman correlation test. Results: The correlation (R) between the VSC and the GF levels was 0.098 with a $p$ value $=0.41 ;-0.084$ with a $p$ value $=0.48$ y 0.067 with a $p$ value $=0.57$ respectively. Conclusions: This study could not demonstrate if there is a relation between the VSC levels and the expression of RANKL and OPG in the GF in patients with chronic periodontitis.
\end{abstract}

Rev. Clin. Periodoncia Implantol. Rehabil. Oral Vol. 6(1); 20-24, 2013.

Key words: Halitosis, periodontitis, volatile sulfur compounds, RANKL and OPG.

\section{INTRODUCCIÓN}

La halitosis, también conocida como mal aliento oral, es un motivo de consulta común en la población ${ }^{(1)}$. La principal causa de halitosis es la microflora oral capaz de producir moléculas volátiles odoríferas como lo son los Compuestos Volátiles Sulfurados (CVS) ${ }^{(2)}$. Aproximadamente un $85 \%$ de las patologías asociadas a la etiología de la halitosis se encuentran en la orofaringe (recubrimiento o placa lingual, gingivitis, periodontitis, amigdalitis, faringitis)(3). Sin embargo, el mal olor bucal también puede originarse por causas extra orales como: Sinusitis, cuerpos extraños en la nariz y enfermedades sistémicas como diabetes, insuficiencia renal, enfermedades hepáticas y gastrointestinales ${ }^{(4-6)}$.

Uno de los métodos más utilizados para el diagnóstico de halitosis es el uso de un monitor de sulfuros portátil (Ejemplo: Halimeter®,
Interscan Corp, USA). Este monitor es relativamente de bajo costo, no requiere de personal entrenado, es portátil y sus mediciones son fáciles y rápidas de realizar. Sin embargo, con este instrumento no es posible diferenciar entre los distintos tipos sulfuros y no puede detectar otra clase de compuestos volátiles ${ }^{(1,7)}$

Por otra parte, la periodontitis es una enfermedad donde se observa una destrucción de los tejidos de soporte del diente que incluyen el ligamento periodontal, el hueso alveolar y el cemento dentario. Claramente, la periodontitis involucra la pérdida irreversible del tejido de inserción periodontal y finalmente la pérdida de la pieza dentaria ${ }^{(8)}$.

Una de las características principales de la pérdida ósea, mediada por un proceso inflamatorio, en periodontitis, es una mayor actividad de los osteoclastos sin un aumento en la formación de hueso. Los osteoclastos son células multinucleadas que derivan del linaje de los

1. Profesor Asistente. Departamento de Periodoncia. Facultad de Odontología, Universidad de los Andes. Chile.

2. Bioquímico. Magíster en Ciencias Biológicas. Centro de Biología y Regeneración Oral (CIBRO). Facultad de Odontología, Universidad de los Andes. Chile. 3. Magíster Salud Pública. Departamento de Ciencias, Investigación y Gestión. Facultad de Odontología, Universidad de los Andes. Chile.

Correspondencia autor: Diego Prieto Damm. djprieto@miuandes.cl. Financiamiento: Esta investigación fue financiada mediante el proyecto FAI INOGTO 2010-25 de la Universidad de los Andes. Trabajo recibido el 30/08/2012. Aprobado para su publicación el 28/01/2013. 
monocitos/macrófagos y son consideradas una de las principales células responsables de la reabsorción ósea. La formación de este tipo celular es impulsada por citoquinas presentes en los tejidos periodontales inflamados y la comprensión de este proceso es fundamental en el desarrollo de estrategias para poder controlarlo( ${ }^{(9)}$.

La reabsorción ósea es regulada por la interacción molecular del receptor activador del factor necrótico $\mathrm{k} \beta$ ligando (RANKL) y osteoprotegerina (OPG). RANKL es expresado predominantemente como un ligando unido a la membrana de osteoblastos, fibroblastos y células $T$ y $B$ activadas $y$ su acción osteoclastogénica puede ser bloqueada por OPG. La expresión de RANKL y OPG está estrechamente regulada por estímulos locales y sistémicos, incluyendo hormonas, mediadores inflamatorios, productos bacterianos y drogas inmunosupresoras. El papel que desempeña la expresión de RANKL y OPG en la regulación de la destrucción ósea, ha sido demostrado en varios estudios in vivo de enfermedades como la artritis bacteriana, artritis reumatoide y periodontitis ${ }^{(10)}$.

En las últimas décadas numerosos estudios han descrito la relación existente entre periodontitis y halitosis. Los CVS se producen por una actividad proteolítica de bacterias periodontales como Porphyromonas gingivalis, Treponema dentícola y Tannerella forsythia. Por otra parte, los CVS parecen estar directamente relacionados con la patogénesis de la enfermedad periodontal. Estos compuestos son capaces de aumentar la permeabilidad de los tejidos periodontales permitiendo la penetración de lipopolisacátidos (LPS) y prostaglandinas ${ }^{(11)}$. También, aumentan la producción de IL-1 y $\mathrm{PGE}_{2}{ }^{(12)}$, activan colagenasas ${ }^{(12)}$, reducen la síntesis de colágeno ${ }^{(13)}$ y causan apoptosis de fibroblastos gingivales humanos ${ }^{(14)}$. Además, suprimen la síntesis de membrana basal y la proliferación de células epiteliales gingivales ${ }^{(15)}$.

La mayoría de los estudios se han enfocados en los efectos que tienen los CVS sobre los tejidos blandos a nivel del periodonto. Recientemente, un estudio realizado in vitro, describió los efectos que podrían tener estos compuestos sobre los tejidos duros mediante la actividad osteoclástica mediada por el sistema RANKL-OPG ${ }^{(16)}$. No existen estudios in vivo que asocien los niveles de CVS y actividad osteoclastogénica medida a través de las concentraciones de RANKL y OPG en el fluido gingival crevicular (FGC). El objetivo de este estudio es evaluar si los CVS medidos en la cavidad oral de pacientes con periodontitis crónica moderada a severa se relacionan con la expresión de RANKL y OPG a nivel de fluido gingival crevicular (FGC).

\section{MÉTODO}

\section{Selección de Pacientes}

Se desarrolló un estudio transversal donde se incluyeron pacientes con periodontitis crónica moderada o severa de ambos sexos entre 25 y 60 años de edad con un mínimo de 12 piezas dentarias presentes en boca. Estos pacientes fueron seleccionados de forma consecutiva y derivados durante los meses de Marzo a Agosto del año 2011 desde la Unidad de Diagnóstico de la Clínica Odontológica Docente Asistencial de la Universidad de los Andes en la comuna de San Bernardo. Se excluyeron pacientes fumadores, que hayan recibido tratamiento con antibióticos, antiinflamatorios o colutorios en los últimos 3 meses, pacientes con periodontitis agresiva o con enfermedades sistémicas que modifiquen la enfermedad periodontal (Ejemplo: Diabetes), pacientes en tratamiento con nifedipino, fenitoína, ciclosporina o anticonceptivos orales y finalmente aquellos que hayan recibido tratamiento periodontal dentro de los últimos 6 meses. Este proyecto fue aprobado por el comité de ética de la Facultad de Odontología de la Universidad de los Andes. Previo al estudio todos los pacientes consintieron voluntariamente en su participación.

\section{Determinación del Estado Periodontal}

Un examinador, previamente calibrado realizó el examen periodontal clínico. Se examinaron todas las piezas dentarias presentes en boca exceptuando los terceros molares. Utilizando una sonda periodontal Carolina del Norte (Hu-Friedy, USA) se midió profundidad al sondaje (PS) y nivel de inserción clínico (NIC) en 6 sitios por cada diente (mesio vestibular, vestibular, disto vestibular, mesio palatino o lingual, palatino o lingual y disto palatino o lingual). Se determinó el índice de sangrado al sondaje (IS) observando las superficies dentarias sangrantes, después de 20 segundos de realizado un recorrido del margen gingival con la sonda periodontal, y promediándolo con las superficies totales examinadas.
Para establecer la definición de caso de periodontitis crónica se utilizó la definición propuesta por el grupo de trabajo CDC (Division of Oral Health at the Centers for Disease Control and Prevention, Atlanta USA), para periodontitis basada en una población de vigilancia(17).

\section{Toma de Muestra de Niveles CVS}

Las muestras de aire para determinar los niveles de CVS fueron obtenidas con una bombilla conectada a la entrada de un monitor de sulfuros (Halimeter ${ }^{\circledR}$, Interscan Corp, USA). Se solicitó a cada paciente que cerraran sus bocas por 30 segundos antes del procedimiento y contuvieran el aliento. Posteriormente, la bombilla se insertó $3 \mathrm{~cm}$ dentro de la boca de cada paciente y se les pidió que sujetaran la bombilla con sus labios y que respiraran solo por la nariz. Los resultados obtenidos se expresaron en partes por billón (ppb).

\section{Toma de Muestra de FGC}

Las nuestras de FGC se obtuvieron de los 2 sacos periodontales más profundos de cada cuadrante con tiras de papel estériles, introduciéndolas subgingivalmente por 30 segundos. Los sitios seleccionados fueron aislados con torundas de algodón, libres de saliva o sangre, y la placa supragingival evidente fue removida cuidadosamente con cureta. Los conos de papel se depositaron en un tubo Eppendorf rotulado y posteriormente las muestras fueron congeladas a $-45^{\circ} \mathrm{C}$ (Freezer ScienTemp, ADRIAN Michigan USA) hasta su análisis.

\section{Test de ELISA para RANKL y OPG}

Las muestras obtenidas del FGC de los pacientes fueron resuspendidas en solución tamponada PBS $1 \mathrm{x}$ y tween 20 al $0.05 \%$ y centrifugadas a 10.000 rpm por 15 minutos a temperatura ambiente. Posteriormente fueron analizadas en el Laboratorio de Investigación Odontológica de la Universidad de los Andes, donde, mediante Test de ELISA, se determinaron los niveles de RANKL y OPG para cada paciente. Para medir los niveles de RANKL se utilizó un kit ELISA de SRANKL humano (BioVendor ${ }$, Austria) y para medir OPG un kit ELISA de Osteoprotegerina humana (RayBioß, USA). El rango de concentraciones de estándares de RANKL era de $0.5,1,2,4,8,16$ y $32 \mathrm{pmol} / \mathrm{l}$ y la de OPG en un rango de concentración de $0 ; 1.23 ; 3.7 ; 11.11 ; 33.33 ; 100$; 300 y $900 \mathrm{pg} / \mathrm{ml}$. Se realizó el análisis según lo indicado por el fabricante. La absorbencia de cada muestra fue cuantificada a $450 \mathrm{~nm}$ en lector de ELISA Microplate Autoreader EL311 (BioTek Instruments $®$, USA).

\section{Análisis Estadístico}

Las variables estudiadas (continuas) se describieron con medidas de tendencia central, posición y dispersión. Se evaluó si las variables analizadas tenían distribución normal a través del test de Shapiro-Wilk.

La relación existente entre los niveles de CVS con los niveles de IS, IG, RANKL, OPG y la razón RANKL/OPG se determinó mediante el test de correlación de Spearman, dado que no presentaron distribución normal y al intentar transformarlas no se logró dicha distribución, presentando su respectivo coeficiente de correlación $(R)$. El análisis fue realizado mediante el software STATA 11.2 (StataCorp LP, Texas, USA). Los resultados obtenidos fueron presentados mediante gráficos de dispersión.

\section{RESULTADOS}

Durante el período en que se realizaron las evaluaciones clínicas y la toma de muestras, fueron evaluados en la clínica de diagnóstico 1014 personas de las cuales $71(7 \%)$ cumplieron todos los criterios de inclusión y exclusión previamente establecidos. 32 pacientes $(45.07 \%)$ fueron de sexo masculino y 39 (54.93\%) de sexo femenino. El promedio de edad fue de 44.83 (Desviación estándar (DE): 10.14). 61 pacientes presentaron $(85.92 \%)$ con periodontitis crónica severa (PCS) y $10(14.08 \%)$ periodontitis crónica moderada (PCM). Las variables analizadas se encuentran descritas en la Tabla 1.

Existió una correlación positiva entre los niveles de CVS y el índice de sangramiento al sondaje (IS) con un R de 0.43 y un $\mathrm{p}$ value $=$ 0.01 (Figura 1). También se observó una correlación positiva entre los niveles de CVS y el IG con un R de 0.309 y un $p$ value $=0.0108$. 
RANKL fue detectado en las muestras de los 71 pacientes, en cambio, OPG fue detectado en 43 pacientes (60.56\%). El valor promedio de RANKL fue de $0.0039 \mathrm{pg} / \mu \mathrm{l}$ (DE: 0.00042) y de OPG fue de $0.02 \mathrm{pg} / \mu \mathrm{l}$ (DE: 0.031).

Al evaluar la correlación de los niveles de CVS con los niveles de RANKL, OPG y la razón de estas, se observó para RANKL un R de 0.098 con un $p$ value $=0.41$, para OPG un $R$ de -0.084 con un $p$ value $=$ 0.48 y para la razón RANKL/OPG un $R$ de 0.067 con un $p$ value $=0.57$ (Figuras 2, 3 y 4 ).

Tabla 1. Descripción de las variables analizadas.

\begin{tabular}{|c|c|c|c|c|}
\hline Variable & $\begin{array}{l}\text { Media } \\
\text { (DE) }\end{array}$ & $\begin{array}{l}\text { Median } \\
(\text { RIC) }\end{array}$ & Mínimo & Máximo \\
\hline Edad & $\begin{array}{l}44.83 \\
(10.14)\end{array}$ & $\begin{array}{l}45 \\
(14)\end{array}$ & 22 & 65 \\
\hline $\mathrm{N}^{\circ}$ de $\mathrm{PD}$ ausentes & & $\begin{array}{l}5 \\
(6)\end{array}$ & 0 & 12 \\
\hline $\mathrm{N}^{\circ}$ de sitios periodontales & $\begin{array}{l}138.08 \\
(20.34)\end{array}$ & $\begin{array}{l}138 \\
(36)\end{array}$ & 96 & 168 \\
\hline $\begin{array}{l}N^{\circ} \text { de sitios con pérdida de NIC }>0= \\
5 \mathrm{~mm}\end{array}$ & $\begin{array}{c}13.41 \\
(12.95)\end{array}$ & $\begin{array}{c}9.52 \\
(16.67)\end{array}$ & 0 & 50 \\
\hline IG & $\begin{array}{c}1.41 \\
(0.42)\end{array}$ & $\begin{array}{c}1.46 \\
(0.18)\end{array}$ & 0.25 & 3 \\
\hline IS (\%) & $\begin{array}{c}57.44 \\
(21.51)\end{array}$ & $\begin{array}{c}60.1 \\
(31.8)\end{array}$ & 9.52 & 93.9 \\
\hline Halimeter $\mathrm{ppb}^{*}$ & $\begin{array}{l}112.53 \\
(82.26)\end{array}$ & $\begin{array}{c}74 \\
(103)\end{array}$ & 17 & 360 \\
\hline RANKL pg/ul & $\begin{array}{c}0.00399 \\
(0.00042)\end{array}$ & $\begin{array}{c}0.00393 \\
(0.00393)\end{array}$ & 0.0033 & 0.0056 \\
\hline 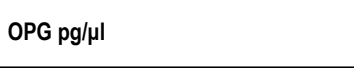 & $\begin{array}{c}0.02 \\
(0.031)\end{array}$ & $\begin{array}{l}0.0077 \\
(0.02)\end{array}$ & 0.0007 & 11.317 \\
\hline Razón RANKL/OPG & $\begin{array}{l}2.51 \\
(2.76)\end{array}$ & $\begin{array}{c}0.51 \\
(5.42)\end{array}$ & 0.02 & 8.02 \\
\hline
\end{tabular}

* Niveles de compuestos volátiles sulfurados.

RIC: Rango intercuartílico.

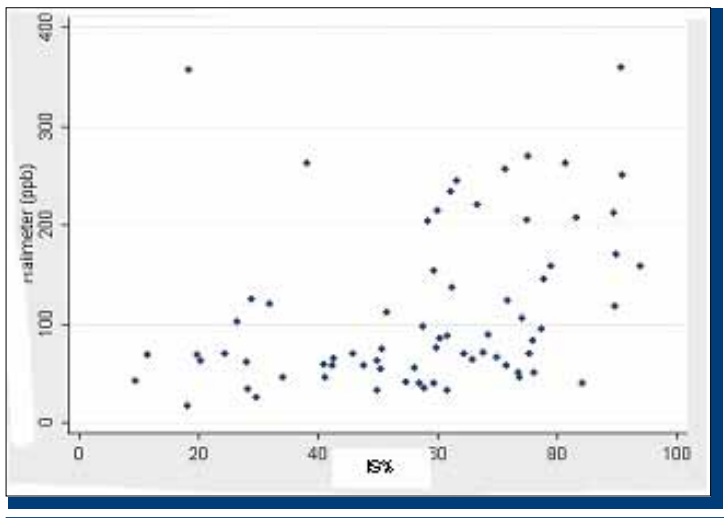

Figura 1. Relación entre los niveles de CVS medidos mediante Halimeter en ppb y el índice de sangramiento al sondaje (IS).

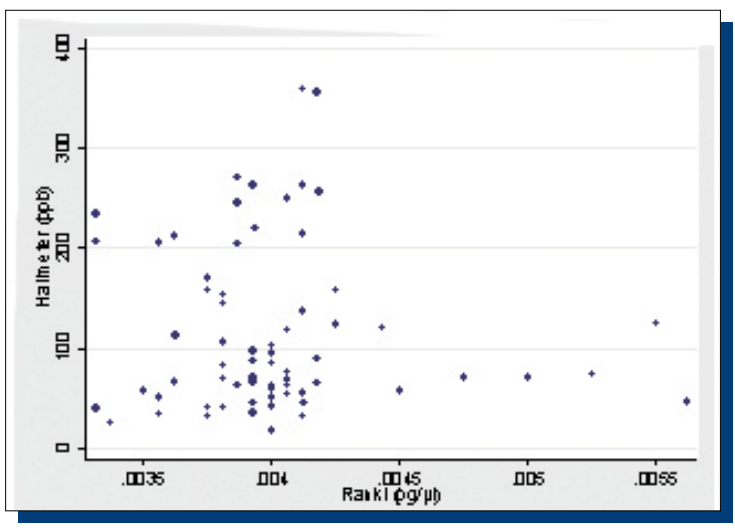

Figura 2. Relación entre los niveles de CVS medidos mediante Halimeter en ppb y RANKL.

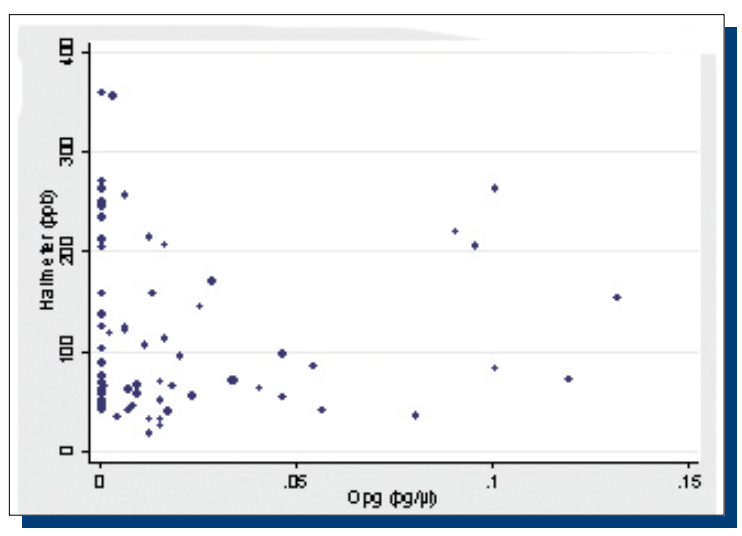

Figura 3. Relación entre los niveles de CVS medidos mediante Halimeter en ppb y OPG.

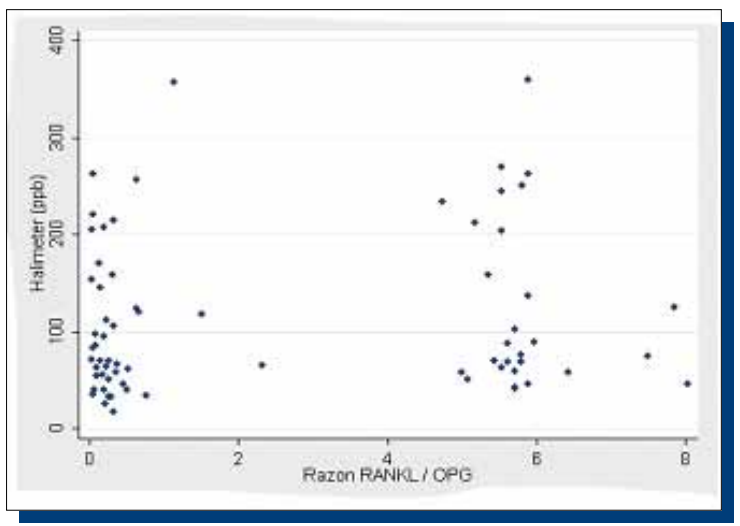

Figura 4. Relación entre los niveles de CVS medidos mediante Halimeter en ppb y la razón $\mathrm{RANKL/OPG}$

\section{DISCUSIÓN}

Existe escasa literatura que describa los efectos de los compuestos volátiles originados en individuos con halitosis de origen intra oral sobre los tejidos periodontales. La gran mayoría de las investigaciones realizadas en los últimos 20 años han sido in vitro. El objetivo de este estudio fue evaluar si los CVS medidos en la cavidad oral de pacientes con periodontitis crónica moderada a severa se relacionan con la expresión de RANKL y OPG a nivel de fluido gingival crevicular (FGC).

El promedio de los niveles de CVS para los pacientes de este estudio fue de $112.53 \mathrm{ppb}$ (DE: 82.26). Podemos comparar estos resultados con los obtenidos por Liu y col. ${ }^{(18)}$, donde en los pacientes con un rango de edad de 35 a 44 años fue de 72 ppb para los hombres y de 86.97 ppb para las mujeres. En el rango de edad de 45 a 54 años fue de $77.33 \mathrm{ppb}$ para los hombres y de 82.53 para las mujeres. El promedio de edad en este estudio fue de $44.83 \pm 10.14$ años. Las diferencias en los niveles de CVS puede deberse a que esta investigación fue realizado en pacientes con periodontitis crónica moderada o severa y el estudio aludido incluyó pacientes con diferentes condiciones periodontales.

Los niveles de RANKL fueron detectados en las muestras de los 71 pacientes evaluados. Vernal y col.(19), encontraron RANKL en el $85 \%$ de pacientes con periodontitis crónica y un $46 \%$ de los controles sanos. El promedio de los niveles de RANKL en esa investigación fue de $115.53 \mathrm{pg}$ (DE: 78.18) en los pacientes con periodontitis crónica y de un 63.08 pg (DE: 55.08) en el grupo de pacientes sanos (al expresar sus resultados en $\mathrm{pg} / \mu \mathrm{l}$ las concentraciones fueron mayores en el grupo de pacientes sanos debido a que la cantidad de fluido gingival crevicular fue considerablemente menor). Estos resultados fueron diferentes a los encontrados en este estudio donde el promedio de RANKL fue de 0.0039 (DE: $0.00042 \mathrm{pg} / \mu \mathrm{l}$ ). Los resultados obtenidos por Vernal y col., demostraron que la cantidad total de RANKL es estadísticamente mayor en sitios activos que en sitios sanos. Esto lo podríamos explicar debido a que esta investigación sólo incluyó pacientes con periodontitis crónica y las diferencias también se podrían deber al kit de ELISA utilizado y su nivel de sensibilidad. 
La halitosis se define como cualquier olor desagradable en el aliento. Se ha descrito que las bacterias responsables de la enfermedad periodontal liberan, producto de su metabolismo, CVS, responsables de la halitosis. Estos compuestos son capaces de producir también cambios a nivel del periodonto, como aumentar la permeabilidad a nivel tisular, estimular la producción de citoquinas inflamatorias, disminuir la síntesis de colágeno e inducir la apoptosis de fibroblastos gingivales. Estudios recientes señalan los posibles efectos que podrían tener los CVS sobre los niveles de RANKL y OPG en el tejido periodontal ${ }^{(11-15,}$ 20-27).

La pérdida de tejido óseo es también una de las manifestaciones de la enfermedad periodontal. Sin embargo, el efecto nocivo de los CVS en metabolismo óseo, en condiciones periodontales, no ha sido totalmente determinado. En un estudio piloto realizado por Irie y col. ${ }^{(16)}$, se examinaron los efectos de NaHS sobre la diferenciación osteoclástica en tejido periodontal de ratas. Después de la aplicación de NaHS, se observó un aumento en la expresión del ARNm de RANKL seguido a un incremento en el número de osteoblastos RANKL positivos y osteoclastos TRAP positivos. Esto sugiere que el sulfuro de hidrógeno puede potencialmente inducir la diferenciación osteoclástica a través de la expresión de RANKL.

Li y col. ${ }^{(28)}$, determinaron que concentraciones fisiológicas de sulfuro de hidrógeno en el aire oral indujeron diferenciación osteoclástica mediante las vías ERK $1 / 2$ y p38 MAPK y que las células multinucleares diferenciadas también tienen la capacidad de producir reabsorción ósea. Así, los CVS, como el sulfuro de hidrógeno, pueden ser un factor causante de reabsorción del hueso alveolar. Imai y col. ${ }^{(29)}$, determinaron que este compuesto volátil también es capaz de inhibir la proliferación de osteoblastos humanos mediante la vía de MAPK.

Una desregulación local en la interacción de RANKL y OPG puede dar lugar a la reabsorción de hueso alveolar. Bostanci y col. ${ }^{(10)}$, analizaron los niveles de RANKL y OPG, así como la razón entre ellos, en pacientes sanos, con gingivitis y diversas formas de periodontitis. Los niveles de RANKL fueron significativamente elevados en todas las formas de periodontitis estudiadas pero no en gingivitis. Estos resultados son similares a los obtenidos en otros estudios que demostraron que los pacientes con periodontitis tienen una mayor expresión de RANKL en comparación con sujetos sanos ${ }^{(19,30,31)}$. Por el contrario, los niveles de OPG se redujeron en todas los grupos con enfermedad periodontal, incluyendo gingivitis. Las alteraciones en las concentraciones de RANKL y OPG reflejaron cambios en la razón de ellos. En comparación con salud, esta relación fue significativamente mayor en todas las formas de periodontitis.

El promedio de los niveles de OPG (0.02 DE: $0.031 \mathrm{pg} / \mathrm{\mu l})$ fue considerable mayor al promedio de los niveles de RANKL (0.0039 DE: $0.00042 \mathrm{pg} / \mathrm{\mu l}$ ) obtenidos. Estos resultados contrastan a los obtenidos por Bostanci y col. ${ }^{(10)}$, donde las muestras de FCG de los pacientes con periodontitis crónica obtuvieron un promedio de $119.6 \mathrm{DE}: 14.4 \mathrm{pg} / \mu \mathrm{l}$ para OPG y de $370.7 \mathrm{DE}: 54.7 \mathrm{pg} / \mu \mathrm{l}$ para RANKL, siendo mayores para esta última citoquina.
En el presente estudio se midieron los niveles de CVS presentes en la boca de los pacientes evaluados. Futuros estudios que determinen los efectos de los CVS sobre los tejidos periodontales deberían ser realizados mediante mediciones hechas en los sacos periodontales.

La enfermedad periodontal progresa de manera impredecible, con periodos de actividad seguidos por periodos de remisión ${ }^{(32)}$. Por este motivo es importante que los clínicos sean capaces de evaluar con precisión si la enfermedad posee sitios activos, la respuesta del hospedero al tratamiento y predecir el curso de la enfermedad en el futuro. Sin embargo, las herramientas actuales no son aplicables en todos los casos. En los años ochenta, Fine y Mandel(33), propusieron usar el sulfuro de hidrógeno como indicador se severidad de enfermedad periodontal. El valor de esa hipótesis aún no se ha dilucidado.

Futuros estudios deberían considerar diferentes estados periodontales como pacientes con gingivitis, periodontitis agresiva y controles sanos. De esta forma se podría determinar de mejor forma la asociación de los CVS sobre la expresión de RANKL y OPG y también se podría usar como grupo control pacientes con todos esos estados periodontales y con bajos niveles de CVS.

Finalmente sería interesante poder determinar si los diferentes compuestos volátiles sulfurados (sulfuro de hidrógeno, metilmercaptano y dimetil sulfuro) tienen una mayor o menor incidencia sobre los tejidos periodontales y la expresión de RANKL y OPG. Esto es importante ya que no todos los pacientes con halitosis tienen las mismas concentraciones de estos gases en la cavidad oral.

Los resultados obtenidos no fueron lo suficientemente robustos para demostrar una asociación estadísticamente significativa entre las variables investigadas. No se pudo apreciar una correlación entre el aumento de los niveles de CVS y el aumento de RANKL y la disminución de OPG. Para demostrar esto, sugerimos la realización de un estudio longitudinal que considere controles sanos, pacientes con diferentes condiciones periodontales y un mayor tamaño de muestra.

\section{AGRADECIMIENTOS}

A la Universidad de los Andes por su apoyo financiero para poder realizar esta investigación.

\section{CONFLICTOS DE INTERÉS} ningún tipo.

\section{REFERENCIAS BIBLIOGRÁFICAS}

1. ADA Council on Scientific Affairs. Oral malodor. J Am Dent Assoc, 2003 Feb; 134(2): 209-214.

2. McNamara TF, Alexander JF, Lee M. The role of microorganisms in the production of oral malodor. Oral Surg Oral Med Oral Pathol, 1972 Jul; 34(1): 41-48.

3. Bazian Ltda. Halitosis. Clin Evid, 2003 Dec; 10: 1640-1643.

4. Preti G, Clark L, Cowart BJ, Feldman RS, Lowry LD, Weber E et al. Non-oral etiologies of oral malodor and altered chemosensation. J Periodontol, 1992 Sep; 63(9): 790-796.

5. Delanghe G, Ghyselen J, van Steenberghe D, Feenstra L. Multidisciplinary breath-odour clinic. Lancet, 1997 Jul 19; 350(9072): 187.

6. Tangerman A, Winkel EG. Extra-oral halitosis: An overview. J Breath Res, 2010 Mar 2; 4(1): 017003.

7. Tanaka M, Anguri H, Nishida N, Ojima M, Nagata H, Shizukuishi S. Reliability of clinical parameters for predicting the outcome of oral malodor treatment. J Dent Res, 2003 Jul; 82(7): 518-522.

8. Kinane DF. Causation and pathogenesis of periodontal disease. Periodontol 2000, 2001; 25: 8-20.

9. Bartold PM, Cantley MD, Haynes DR. Mechanisms and control of pathologic bone loss in periodontitis. Periodontol 2000, 2010 Jun; 53: 55-69.

10. Bostanci N, Ilgenli T, Emingil G, Afacan B, Han B, Toz H et al. Gingival crevicular fluid levels of RANKL and OPG in periodontal diseases: Implications of their relative ratio. J Clin Periodontol, 2007 May; 34(5): 370-376.

11. Ng W, Tonzetich J. Effect of hydrogen sulfide and methyl mercaptan on the permeability of oral mucosa. J Dent Res, 1984 Jul; 63(7): 994-997.

12. Ratkay LG, Waterfield JD, Tonzetich J. Stimulation of enzyme and cytokine production by methyl mercaptan in human gingival fibroblast and monocyte cell cultures. Arch Oral Biol, 1995 Apr; 40(4): 337-344.
13. Johnson P, Yaegaki K, Tonzetich J. Effect of methyl mercaptan on synthesis and degradation of collagen. J Periodontal Res, 1996 Jul; 31(5): 323-329.

14. Yaegaki K, Qian W, Murata T, Imai T, Sato T, Tanaka T et al. Oral malodorous compound causes apoptosis and genomic DNA damage in human gingival fibroblasts. J Periodontal Res, 2008 Aug; 43(4): 391-399.

15. Takeuchi H, Setoguchi T, Machigashira M, Kanbara K, Izumi Y. Hydrogen sulfide inhibits cell proliferation and induces cell cycle arrest via an elevated p21 Cip1 level in Ca9-22 cells. J Periodontal Res, 2008 Feb; 43(1): 90-95.

16. Irie K, Ekuni D, Yamamoto T, Morita M, Yaegaki K, li H et al. A single application of hydrogen sulphide induces a transient osteoclast differentiation with RANKL expression in the rat model. Arch Oral Biol, 2009 Aug; 54(8): 723-729.

17. Page RC, Eke PI. Case definitions for use in population-based surveillance of periodontitis. J Periodontol, 2007 Jul; 78(7 Suppl): 1387-1399.

18. Liu XN, Shinada K, Chen XC, Zhang BX, Yaegaki K, Kawaguchi Y. Oral malodorrelated parameters in the chinese general population. J Clin Periodontol, 2006 Jan; 33(1): 31-36.

19. Vernal R, Chaparro A, Graumann R, Puente J, Valenzuela MA, Gamonal J. Levels of cytokine receptor activator of nuclear factor kappaB ligand in gingival crevicular fluid in untreated chronic periodontitis patients. J Periodontol, $2004 \mathrm{Dec}$ 75(12): 1586-1591.

20. Murata T, Yaegaki K, Qian W, Herai M, Calenic B, Imai T et al. Hydrogen sulfide induces apoptosis in epithelial cells derived from human gingiva. $J$ Breath Res, 2008 Mar; 2(1): 017007

21. Calenic B, Yaegaki K, Kozhuharova A, Imai T. Oral malodorous compound causes oxidative stress and p53-mediated programmed cell death in keratinocyte stem cells. J Periodontol, 2010 Sep; 81(9): 1317-1323.

22. Calenic B, Yaegaki K, Murata T, Imai T, Aoyama I, Sato T et al. Oral malodorous 
compound triggers mitochondrial-dependent apoptosis and causes genomic DNA damage in human gingival epithelial cells. J Periodontal Res, 2010 Feb; 45(1): 31-37.

23. Morita M, Wang HL. Relationship between sulcular sulfide level and oral malodor in subjects with periodontal disease. J Periodontol, 2001 Jan; 72(1): 79-84.

24. Ratcliff PA, Johnson PW. The relationship between oral malodor, gingivitis, and periodontitis. A review. J Periodontol, 1999 May; 70(5): 485-489.

25. Zhang JH, Dong Z, Chu L. Hydrogen sulfide induces apoptosis in human periodontium cells. J Periodontal Res, 2010 Feb; 45(1): 71-78.

26. Zhi L, Ang AD, Zhang H, Moore PK, Bhatia M. Hydrogen sulfide induces the synthesis of proinflammatory cytokines in human monocyte cell line U937 via the ERK-NF-kappaB pathway. J Leukoc Biol, 2007 May; 81(5): 1322-1332.

27. Persson S, Edlund MB, Claesson R, Carlsson J. The formation of hydrogen sulfide and methyl mercaptan by oral bacteria. Oral Microbiol Immunol, 1990 Aug; 5(4): 195-201.

28. li H, Imai T, Yaegaki K, Irie K, Ekuni D, Morita M. Oral malodorous compound induces osteoclast differentiation without receptor activator of nuclear factor kappaB ligand. J Periodontol, 2010 Nov; 81(11): 1691-1697.
29. Imai T, li H, Yaegaki K, Murata T, Sato T, Kamoda T. Oral malodorous compound inhibits osteoblast proliferation. J Periodontol, 2009 Dec; 80(12): 2028-2034

30. Mogi M, Otogoto J, Ota N, Togari A. Differential expression of RANKL and osteoprotegerin in gingival crevicular fluid of patients with periodontitis. J Dent Res, 2004 Feb; 83(2): 166-169.

31. Lu HK, Chen YL, Chang HC, Li CL, Kuo MY. Identification of the osteoprotegerin receptor activator of nuclear factor-kappa $B$ ligand system in gingival crevicular fluid and tissue of patients with chronic periodontitis. J Periodontal Res, 2006 Aug; 41(4) 354-360.

32. Socransky SS, Haffajee AD, Goodson JM, Lindhe J. New concepts of destructive periodontal disease. J Clin Periodontol, 1984 Jan; 11(1): 21-32.

33. Fine $\mathrm{DH}$, Mandel ID. Indicators of periodontal disease activity: An evaluation. $J$ Clin Periodontol, 1986 May; 13(5): 533-546. 